\title{
Минимальные личевые
}

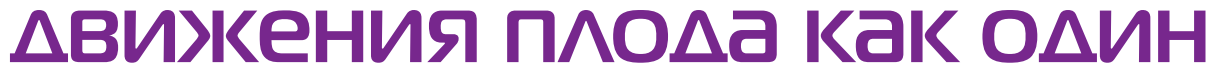 \\ из критериев Аиагностики внутриутробного Аистресса}

\author{
Коротаева Н.В. ${ }^{1,2}$, \\ Ипполитова $\Lambda$. И. $^{2}$, \\ Федотова Т.В. ${ }^{1}$, \\ Чибисова Н.А. ${ }^{1}$, \\ Першина E.C. ${ }^{2}$
}

\begin{abstract}
1 Перинатальный центр, Бюджетное учреждение здравоохранения Воронежской области “Воронежская областная клиническая больница № 1", 394066 , г. Воронеж, Российская Федерация

2 Федеральное государственное бюджетное образовательное учреждение высшего образования «Воронежский государственный медицинский университет имени Н.Н. Бурденко" Министерства зАравоохранения Российской Федерации, 394036, г. Воронеж, Российская Федерация
\end{abstract}

Использование оценки лицевых движений плода в качестве дополнительного критерия развития возможного дистресса является одним из перспективных направлений в современной неонатологии, что связано с тем фактом, что плоды с 20-22-й по 28-ю неделю гестации являются уязвимой группой по развитию дистрессовых состояний ввиду невозможности использования у них кардиотокографии (КТГ).

Цель исследования - оценка состояний дистресса у плодов в сроке с 22-24 нед гестации с применением стандартизированной совокупности лицевых кодов мимики новорожденных при ультразвуковой (УЗ) диагностике в разрешении 3D и 4D.

Материал и методы. В исследование были включены 313 плодов в сроке гестации не менее 2224 нед, проходивших консультацию и УЗ-диагностику на базе БУЗ В0 «Воронежская областная клиническая больница № 1 “Перинатальный центр”» с 2017 по 2019 г. Все исследуемые плоды были разделены на 2 группы: 1-я группа - с диагностированными случаями дистресса в акушерском анамнезе $(n=98)$, 2-я группа - с отсутствием клинических признаков дистресса $(n=215)$. Основными критериями включения плодов в 1-ю группу исследования являлось наличие одного или нескольких критериев: тахикардия/ брадикардия; стойкая монотонность ритма (генерация записи 5 уд/мин и меньше); ранние, вариабельные и поздние децелерации с амплитудой более 30 уд/мин. Все плоды, принявшие участие в исследовании, были обследованы с помощью аппарата GE Healthcare Voluson 730. Отдельно хочется отметить, что плоды с гестационным возрастом более 32 нед, имеющие вышеперечисленные клинические признаки дистресса, были обследованы на той же аппаратуре сразу после проведения КТГ.

Результаты и обсуждение. В ходе исследования особое внимание было уделено последовательной совокупности лицевых движений плода, где важными диагностическими (и в последующем прогностическими) признаками у исследуемых являлись опускание брови, поднятие внутренней части брови, поднятие внешней части брови и сближение внутренних углов бровей. Данная последовательность в момент дистресса была оценена нами как фундаментальная комбинация действий на лице плода.

Заключение. Полученные сравнительные данные свидетельствуют о том, что развивающееся направление «чтение эмоций» является перспективным в ранней диагностике дистресса плода. Оценка лицевых движений, позволяющая выявить дистрессовое состояние, требует дальнейшего изучения и популяризации, так как не влечет за собой дополнительных материальных затрат и может быть использована для применения в уязвимой группе плодов (неприемлем метод кардиотокографии) с 20-22-й по 28-ю неделю гестации.

Финансирование. Исследование не имело финансовой поддержки.

Конфликт интересов. Авторы заявляют об отсутствии конфликта интересов.

Для цитирования: Коротаева Н.В., Ипполитова Л.И., Федотова Т.В., Чибисова Н.А., Першина Е.С. Минимальные лицевые движения плода как один из критериев диагностики внутриутробного дистресса // Неонатология: новости, мнения, обучение. 2020. T. 8, № 2. С. 7-12. D0I: 10.33029/2308-2402-2020-8-2-7-12

Статья поступила в редакцию 06.10.2019. Принята в печать 04.06.2020
Ключевые слова: неонатология, недоношенные дети, дистресс плода, лицевые движения, УЗ-диагностика 
Minimal facial movements of the fetus as one of the diagnostic criteria intrauterine distress

Korotaeva N.V.1,, Ippolitova L.I. ${ }^{2}$,

Fedotova T.V. ${ }^{1}$, Chibisova N.A. ${ }^{1}$

Pershina E.S. ${ }^{2}$
${ }^{1}$ Perinatal Center, Voronezh Regional Clinical Hospital No. 1,

394066, Voronezh, Russian Federation

${ }^{2}$ N.N. Burdenko Voronezh State Medical University of the Ministry of Healthcare of the Russian Federation, 394036, Voronezh, Russian Federation

The use of the facial movements' evaluation of the fetus as an additional criterion for the detection of the possible fetal distress development is one of the promising trends in modern neonatology, since fetuses from 20-22 to 28 weeks of gestation are a vulnerable group for the development of distress conditions due to the impossibility of using cardiotocography among them.

The aim of the study is to assess the conditions of distress in fetuses in the period from 22-24 weeks of gestation using a standardized set of facial expressions of newborn children in ultrasound diagnostics in $3 \mathrm{D}$ and $4 \mathrm{D}$ resolution.

Material and methods. The study included 313 fetuses in the gestation period of at least 22-24 weeks, which were consulted and ultrasound-diagnosed in Voronezh Regional Clinical Hospital No.1 "Perinatal Center" in the period of 2017-2019. All the fetuses were divided into 2 groups: the $1^{\text {st }}$ group - fetuses with diagnosed cases of distress in obstetric history $(n=98)$, and the $2^{\text {nd }}$ group - fetuses with no clinical signs of distress $(n=215)$. The main criteria for the inclusion of fetuses in the $1^{\text {st }}$ group of the study were the presence of one or more signs: tachycardia/bradycardia; persistent monotonous of rhythm (recording of $5 \mathrm{bpm}$ or less); early, variable and late deceleration with amplitude of more than 30 beats/min. All fetuses with clinical signs of distress mentioned above were examined immediately after CTG by GE Healthcare Voluson 730.

Results and discussions. During the study, special attention was given to the consistent sets of facial movements of the fetus, where important diagnostic (and subsequent predictive feature) traits were lowering eyebrows, raising inner part of the eyebrow, raising outer part of the eyebrow, and converging inner corners of the eyebrows. This sequence at the time of distress was evaluated by us as a fundamental combination of actions on the face of the fetus.

Conclusions. The comparative data obtained show that the "reading of emotions" is promising in the early diagnosis of "fetal distress". Evaluation of the facial movements of the fetus, which allows to detect the fetal distress needs to be examined and popularized further, since it does not include any additional material costs and can be used in a vulnerable group of fetuses (unacceptable CTG method) from 20-22 to 28 weeks of gestation.

Funding. The study had no sponsor support.

Conflict of interests. The authors declare no conflict of interests.

For citation: Korotaeva N.V., Ippolitova L.I., Fedotova T.V., Chibisova N.A., Pershina E.S. Minimal facial movements of the fetus as one of the diagnostic criteria intrauterine distress. Neonatologiya: novosti, mneniya, obuchenie [Neonatology: News, Opinions, Training]. 2020; 8 (2): 7-12. D0I: 10.33029/2308-2402-2020-8-2-7-12 (in Russian)

Received 06.10.2019. Accepted 04.06.2020.

И сторически термин «дистресс» в акушерстве и неонатологии использовался для описания случаев, когда плод не получал достаточного количества кислорода во время беременности или родов [1]. Особое внимание к данному состоянию в современной практике врачей не случайно: оно связано с последствиями и физиологическими перестройками внутри организма плода, которые могут привести к негативным последствиям.

Известно, что при снижении уровня кислорода плод испытывает 3 стадии: преходящую гипоксию без метаболического ацидоза, тканевую гипоксию с риском метаболического ацидоза и гипоксию с развившимся метаболическим ацидозом [2]. Все эти реакции на кислородную недостаточность регулируются автономной нервной системой, опосредован-

\section{Keywords:} neonatology, premature infants, fetal distress, facial movements, ultrasound diagnosis ной каскадными парасимпатическими и симпатическими механизмами. Компенсаторные механизмы, направленные на поддержание плода во время родов, к сожалению, не могут помочь в случае длительной непрерывной гипоксии плода. Таким образом, последнее состояние приводит к снижению частоты сердечных сокращений, перераспределению сердечного выброса преимущественно перфузирующим органам, таким как сердце, мозг и надпочечники, и переходу к анаэробному клеточному метаболизму [3]. Все эти каскадные реакции связаны со значительной перинатальной заболеваемостью и смертностью, при этом особое внимание уделяется краткосрочным и долгосрочным осложнениям, включая энцефалопатию, судороги, церебральный паралич и нарушения психоречевого и моторного развития $[4,5]$. 
Тот факт, что частота сердечных сокращений плода заметно изменяется в ответ на длительную кислородную недостаточность, делал мониторинг данного параметра ценным и широко используемым инструментом для диагностики дистресса плода в течение многих лет. С развитием методов диагностики и новейших подходов перед врачами разных специальностей открылись новые возможности ранней диагностики фетального дистресса, к которым относится кардиотокография (КТГ), что существенно увеличило раннюю диагностику и своевременную коррекцию данного состояния.

Несмотря на то что КТГ является «золотым стандартом» в диагностике дистресса у плода во внутриутробном периоде, использование данного метода ограничено гестационным возрастом исследуемых: плоды с 20-22-й по 28-ю неделю гестации являются уязвимой группой по угрозе развития дистрессовых состояний вследствие невозможности применения у них метода КТГ. Учитывая данный факт, одним из перспективнейших и развивающихся направлений в ранней диагностике дистресса плода можно смело считать оценку лицевых моделей поведения, что имеет под собой анатомические и пренатальные обоснования.

Доказано, что лицо плода опосредует собой реакции мозга на изменения, касающиеся центральной нервной системы и организма в целом $[6,7]$. Лицевой и тройничный нервы, иннервирующие мышцы мимики лица, появляются на сроке 10-11 нед гестации, а иннервируемые мышцы к 16-й неделе [8]. Несмотря на раннее появление нервов и иннервируемых ими мышц в онтогенезе, полная оценка их совместной неврологической деятельности во внутриутробном периоде разумна только с 22-24-й недели гестации, что связано с развитием жировой ткани на лице плода, которое продолжается вплоть до 36-й недели беременности.

Таким образом, использование оценки лицевых движений плода в качестве дополнительного критерия развития возможного дистресса является одним из перспективных направлений в современных неонатологии, акушерстве и гинекологии.

Цель исследования - оценка состояний дистресса у плодов в сроке с 22-24 нед гестации с применением стандартизированной совокупности лицевых кодов мимики новорожденных при У3-диагностике в разрешении 3D и 4D.

\section{Материал и методы}

В исследование были включены 313 плодов в сроке гестации не менее 22-24 нед, проходивших консультацию и УЗ-диагностику на базе БУЗ В0 «Воронежская областная клиническая больница № 1 “Перинатальный центр"» с 2017 по 2019 г. Исследователями было получено добровольное информированное согласие на участие в исследовании у законных представителей. Все исследуемые плоды были разделены на 2 группы: 1-я группа - с диагностированными случаями дистресса в акушерском анамнезе $(n=98)$, 2-я группа - с отсутствием клинических признаков дистресса (n=215). Для верификации диагноза дистресса КТГ проводили с 32-й недели гестации всем исследуемым. КТГ оценивали согласно критериям FIGO (2015): базальная частота сердечных сокращений (ЧСС) плода, вариабельность базального ритма и децелерации. В соответствие с полученными результатами лента КТГ расценивалась специалистом как нормальная, подозрительная или патологическая.

Основными критериями включения плодов в 1-ю группу исследования являлось наличие одного или нескольких критериев, согласно данным КТГ: тахикардия/брадикардия; стойкая монотонность ритма (генерация записи 5 уд/мин и меньше); ранние, вариабельные и поздние децелерации с амплитудой >30 уд/мин. Допплерометрия проводилась с 24-й недели гестации всем плодам, принимавшим участие в исследовании. В качестве проявлений дистресса специалисты рассматривали следующие параметры: снижение скорости в диастолу (ниже нормативных показателей), повышение пульсационного индекса в маточной артерии, регистрация дикротической выемки (в одной/обеих маточных артериях). Все плоды, имеющие клинические признаки дистресса согласно вышеперечисленным критериям, сразу после проведения КТГ и допплерометрии были обследованы с помощью аппарата УЗ-диагностики, при проведении которой с разрешения законных представителей получено согласие на фото- и видеофиксацию материалов. При проведении У3-диагностики использовали аппарат GE Healthcare Voluson 730 и брюшной датчик 5 МГц. Критерием выбора данного аппарата в исследовании являлась его техническая возможность съемки от 15 до 24 фотограмм в секунду (в зависимости от заданного диапазона углов). При исследовании минимальных мимических единиц на GE Healthcare Voluson 730 датчик был расположен специалистом таким образом, чтобы на экран выводилась сагиттальная часть лица плода, включающая лоб, нос и рот - это было необходимо для оценки анализа поведения плода. Окончательная верификация диагноза основывалась на оценке по шкале Апгар на 1-й и 5-й минутах жизни (баллы) и среднем уровне лактата (ммоль/л).

\section{Результаты и обсужкение}

Подходя к оценке дистресса у плодов и новорожденных, стоит понимать, что данное состояние охватывает не только физическую, но и психологическую стороны боли и страдания, что неоднократно подтверждено исследованиями [9-11]. При оценке лицевых движений плодов сложность ранней диагностики неразрывно связана с тем, что выражения лица и минимальные мимические единицы не всегда могут соответствовать стереотипным выражениям отрицательных эмоций, представляя собой группу лицевых конфигураций, которые довольно многообразны и изменчивы по своей морфологии.

Согласно исследованиям, корковые зоны у недоношенных детей активируются болевыми раздражителями и различаются при сравнении ответов на внутриутробные раздражения [12]. Термин «боль/дистресс гештальт» применим к эмоциональным выражениям, которые становятся координированными со временем в ответ на дистрессовые состояния по мере «созревания» ноцицептивной нервной системы, что нашло отражение в зарубежных исследованиях [13]. 

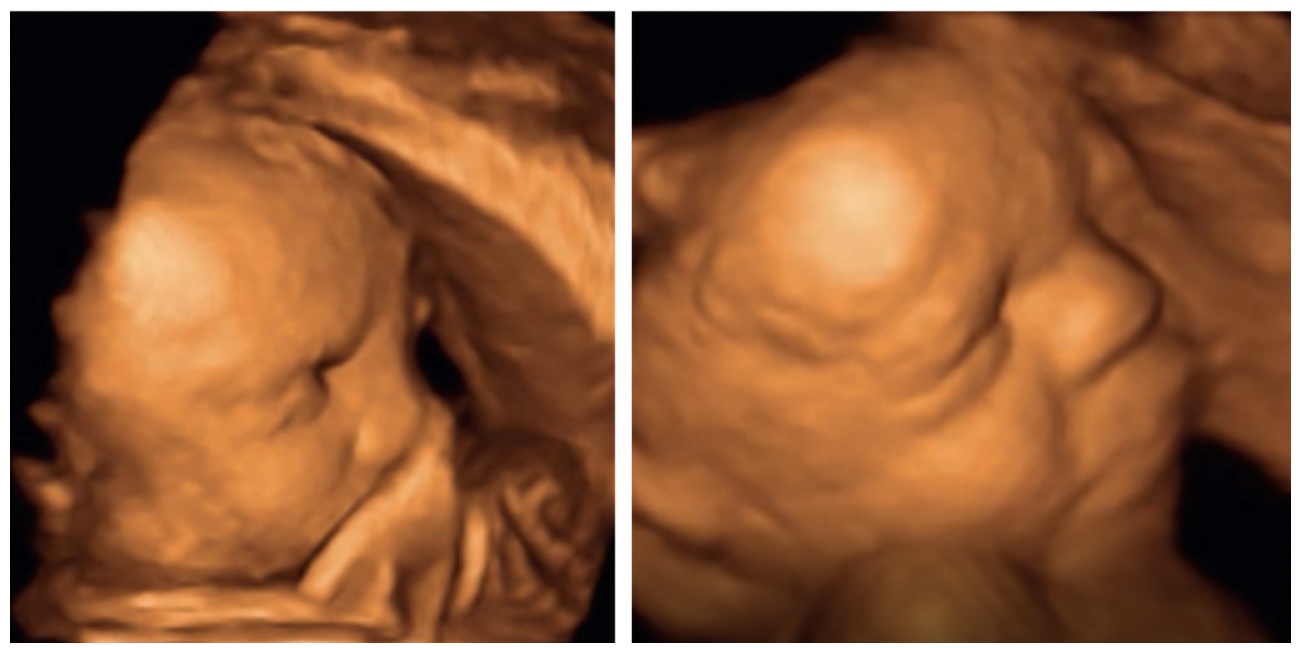

Рис. 1. Пример опускания бровей у плодов со сроком гестации 27 неА при ультразвуковом исследовании в разрешении 3D

В ходе исследования особое внимание было уделено не наличию отдельных известных лицевых движений плода, а их последовательной совокупности, где важными диагностическим (и в последующем прогностическим) признаком являлись опускание брови, поднятие внутренней и внешней части брови и сближение внутренних углов бровей. Данная последовательность в момент дистресса была оценена нами как фундаментальная комбинация действий на лице плода.

Выделение нами опускания брови как пускового и обязательного предиктора возможного дистресса неслучайно: данное лицевое движение отдельно (или в сочетании с другими известными двигательными лицевыми единицами) является обязательным компонентом отрицательных эмоций у детей и никогда не встречается при положительных эмоциях [14] (рис. 1).

В дополнение к опусканию брови (при вовлечении musculus procerus, musculus depressor supercilii и musculus corrugator supercilii) нами были оценены и детально проанализированы другие паттерны лица, которые связаны с негативной эмоциональной реакцией у новорожденных, следующей друг за другом [14]: поднятие внутренней части брови (при вовлечении медиальной части musculus epicranius), поднятие внешней части брови (при вовлечении латеральной части musculus epicranius) и сближение внутренних углов бровей (рис. 2).

Для подтверждения достоверности наличия выделенных лицевых движений у плодов в состоянии дистресс-синдрома была оценена встречаемость последовательности мимических единиц у плодов в сроке гестации от 28 до 37 нед с наличием подтвержденного дистресс-синдрома и без него по данным КТГ. КТГ проводилась всем исследуемым в непрерывном режиме при участии специалиста, владеющего данной процедурой.

При оценке комбинации действий на лице плодов, имеющих подтвержденный дистресс-синдром, было выявлено, что
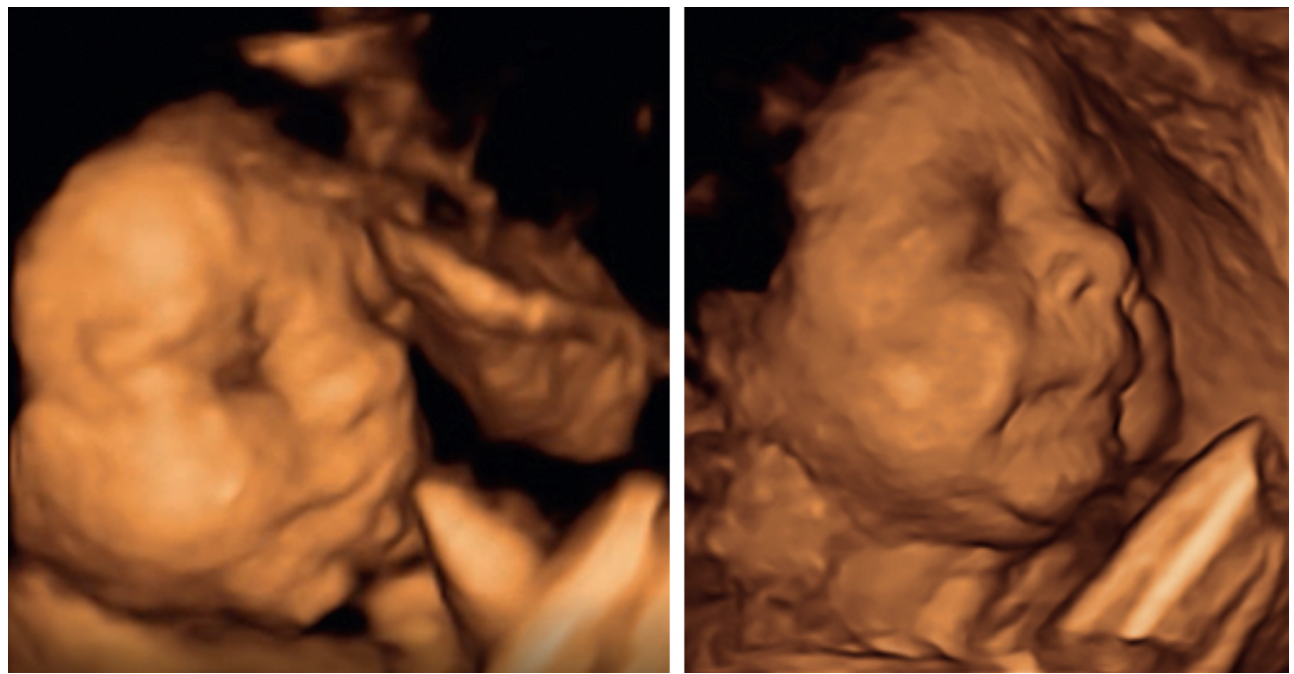

Рис. 2. Пример поднятия внутренней и внешней частей брови и сближения внутренних углов у плодов со сроком гестации 24 неА при ультразвуковом исслеАовании в разрешении 3D 

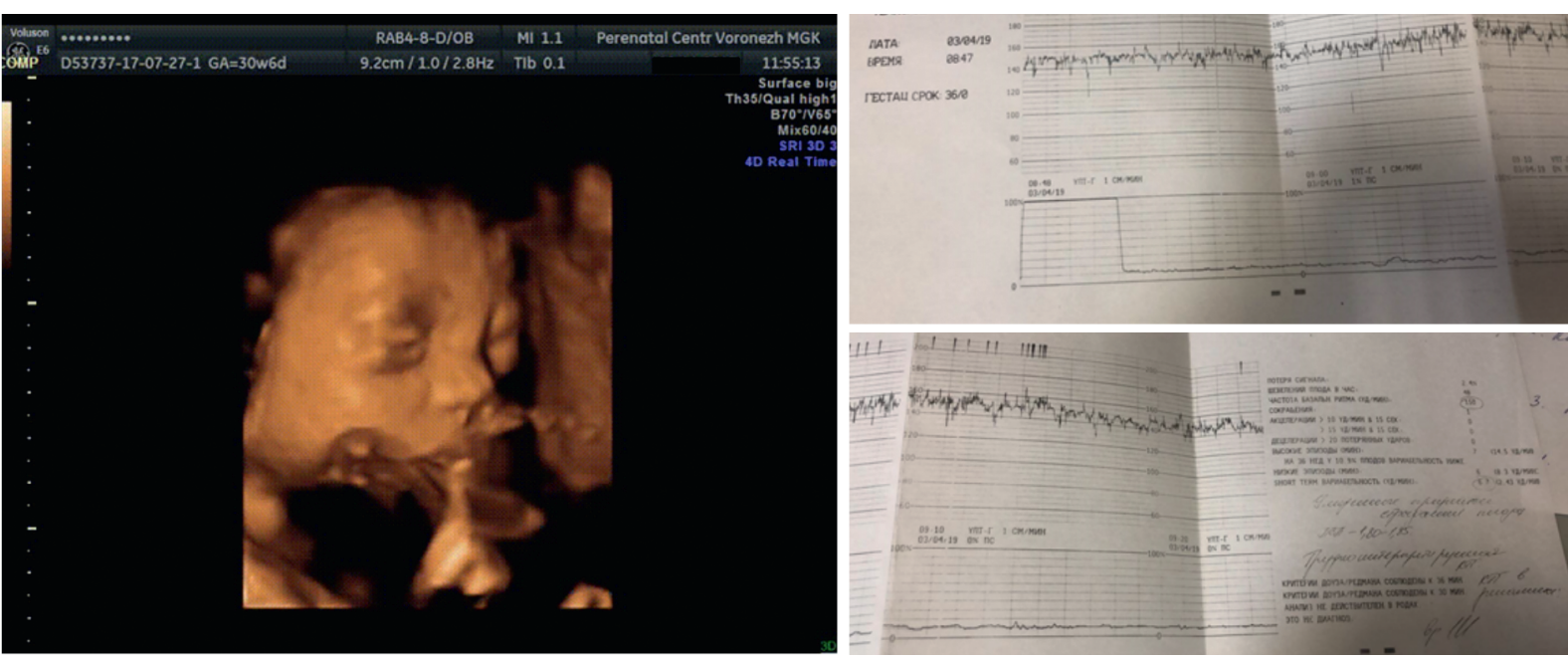

Рис. 3. Пример последовательности мицевых Авигательных еАиниц с подтвержденным состоянием страдания плода в сроке гестации 36 нед по данным ультразвуковой диагностики и кардиотокографии

отдельно такая лицевая двигательная единица, как опускание брови, встречалась в 98,9\% случаев, поднятие внутренней части брови - в 96,9\%, поднятие внешней части брови в 96,9\%, сближение внутренних углов бровей - в 97,9\%. Комбинация 4 представленных показателей в единицу времени встречалась в 96,9\% у плодов с подтвержденным дистресссиндромом по данным КТГ (рис. 3). При анализе оценки по шкале Апгар на 1-й и 5-й минутах жизни было выявлено, что в группе обследуемых с диагностированным дистрессом

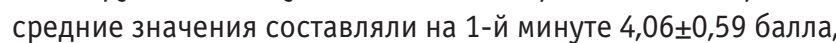
на 5-й минуте 5,78 $\pm 0,47$ балла, средний уровень лактата 6,3 $\pm 1,7$ ммоль/л ( $\mathrm{pH} 7,28-7,23, \mathrm{BE}-10-5,6)$.

При оценке комбинации действий на лице плодов, не имеющих подтвержденного дистресс-синдрома, было выявлено, что отдельно такая лицевая двигательная единица, как опускание брови, встречалась в 2,3\% случаев, поднятие внутренней части брови - в 1,39\%, поднятие внешней части брови - в 1,39\%, сближение внутренних углов бровей в 2,79\%. Комбинация 4 представленных показателей в единицу времени не встречалась ни у одного из плодов, не имеющих дистресс-синдром по данным КТГ. Данное исследование легло в основу патента на изобретение № 2019114714/ 14 (028141).

\section{Зак^ючение}

Полученные сравнительные данные демонстрируют тот факт, что развивающееся направление «чтение эмоций» перспективно в ранней диагностике дистресса плода и может быть использовано для применения в уязвимой группе плодов с 20-22-й по 28-ю неделю гестации. Авторы понимают, что оценка лицевых движений плода, позволяющих выявить дистрессовое состояние, требует дальнейшего изучения и исследований в данной области.

\section{CBEAEНИЯ ОБ АВTOPAX}

Коротаева Наталья Владимировна (Natalia V. Korotaeva) - кандидат медицинских наук, врач-неонатолог Перинатального центра БУЗ В0 ВОКБ № 1, ассистент кафедры госпитальной и поликлинической педиатрии ФГБОУ ВО ВГМУ им. Н.Н. Бурденко Минздрава России, Воронеж, Российская Федерация

E-mail: korotaeva.nv@mail.ru

https://orcid.org/0000-0001-5859-7717

Ипполитова Людмила Ивановна (Lyudmila I. Ippolitova) - главный внештатный неонатолог Воронежской области, профессор кафедры госпитальной и поликлинической педиатрии ФГБОУ ВО ВГМУ им. Н.Н. Бурденко Минздрава России, Воронеж, Российская Федерация

E-mail: liippolitova@okb-vrn.ru

https://orcid.org/0000-0001-7076-0484

Федотова Татьяна Валерьевна (Tatyana V. Fedotova) - врач-генетик высшей категории, заведующая медико-генетической консультацией Перинатального центра БУЗ В0 ВОКБ № 1, Воронеж, Российская Федерация

E-mail: mail@vokb1.zdrav36.ru

https://orcid.org/0000-0002-4469-2374

Чибисова Надежда Александровна (Nadezhda A. Chibisova) - врач ультразвуковой диагностики Перинатального центра БУ3 В0 ВОКБ № 1, Воронеж, Российская Федерация

E-mail: mail@vokb1.zdrav36.ru

https://orcid.org/0000-0001-5782-5861 
Першина Елена Сергеевна (Elena S. Pershina) - ординатор-неонатолог кафедры госпитальной и поликлинической педиатрии ФГБОУ ВО ВГМУ им. Н.Н. Бурденко Минздрава России, Воронеж, Российская Федерация

E-mail: pershina.elenasergeevna@gmail.com

https://orcid.org/0000-0003-2182-1062

\section{^ИTEPATYPA/REFERENCES}

1. Mccall J.O. Jr, Fulsher R.W. A study of fetal distress, its interpretation and significance. Am J Obstet Gynecol. 1953; 65 (5): 1006-19.

2. Martin C.B. Jr. Normal fetal physiology and behavior, and adaptive responses with hypoxemia. Semin Perinatol. 2008; 32: 239-42.

3. Fahey J., King T.L. Intrauterine asphyxia: clinical implications for providers of intrapartum care. J Midwifery Womens Health. 2005; 50: 498-506.

4. Executive summary: neonatal encephalopathy and neurologic outcome, second edition, report of the American College of Obstetricians and Gynecologists' Task Force on Neonatal Encephalopathy. Obstet Gynecol. 2014; 123: 896-901.

5. Hankins G.D., Speer M. Defining the pathogenesis and pathophysiology of neonatal encephalopathy and cerebral palsy. Obstet Gynecol. 2003; 102: 628-36.

6. Prechtl H.F. Qualitative changes of spontaneous movements in fetus and preterm infant are a marker of neurological dysfunction. Early Hum Dev. 1990; 23 (3): 151-8.

7. Prechtl H.F. State of the art of a new functional assessment of the young nervous system: an early predictor of cerebral palsy. Early Hum Dev 1997; 50 (1): 1-11.
8. Kurjak A., Azumendi G., Andonotopo W., Salihagic-Kadic A Three- and four-dimensional ultrasonography for the structural and functional evaluation of the fetal face. Am J Obstet Gynecol 2007; 196 (1) 16-28.

9. Sroufe A. Socioemotional development. In J.D. Osofsky (ed.). Handbook of Infant Development. New York: Wiley, 1979: 462-516.

10. Oster H., Hegley D., Nagel L. Adult judgements and fine-grained analysis of infant facial expressions: Testing the validity of a priori coding formulas. Dev Psychol. 1992; 28: 1115-31.

11. Young G., Décarie T.G. An ethology-based catalogue of facial-vocal behaviour in infancy. Anim Behav. 1977; 25: 95-107.

12. Bartocci M., Bergqvist L.L., Lagercrantz H., Anand K.J. Pain activates cortical areas in the preterm newborn brain. Pain. 2006; $122(1-2)$ 109-17.

13. Reissland N., Francis B., Mason J. Can healthy fetuses show facial expressions of "pain" or "distress"? PLoS One. 2013; 8 (6): e65530.

14. Oster H. Baby FACS: Facial Action Coding System for Infants and Young Children. Unpublished monograph and coding manual. New York: New York University, 2009 